\title{
Comportamento do consumidor em relação a produtos sustentáveis: uma revisão sistemática de literatura
}

\author{
Luciana Faluba Damázio, Leonardo Antunes Nogueira Coutinho e Helena \\ Belintani Shigaki
}

\section{RESUMO}

Este artigo tem por objetivo realizar uma revisão sistemática de literatura sobre o comportamento do consumidor em relação aos produtos sustentáveis, a fim de identificar os fatores que interferem no comportamento, bem como definir os atributos e a percepção do consumidor frente ao tema sustentabilidade. Identificou-se mais de 4600 textos a partir da revisão, conduzida em 22 bases acadêmicas e practitioners. A partir dos critérios de inclusão e exclusão, 219 artigos foram selecionados e, desses, 95 foram totalmente analisados. Como resultado, foram identificados onze fatores de influência nas práticas de sustentabilidade no comportamento do consumidor de produtos sustentáveis (ambiente, idade, nível educacional, classe econômica, gênero, consciência, preocupação com a própria imagem, emoção, acesso à informação, confiança e hábito) e sete em relação aos atributos e percepção do consumidor acerca de sustentabilidade (eco-friendly, garantia de origem, saúde do consumidor, fair trade, teste em animais, recicláveis e ingredientes naturais).

Palavras-chave: consumo sustentável; comportamento do consumidor; sustentabilidade; atributo

Consumer behavior on sustainable products: a systematic review of literature

\section{ABSTRACT}

This paper aims to conduct a systematic review of the literature on consumer behavior about sustainable products, in order to identify the factors that interfere in consumer behavior, as well as define sustainability attributes and the perception of the consumer about sustainability. More than 4600 texts were identified from a review, conducted in 22 academic and practitioners' bases. Based on inclusion and exclusion criteria, 219 papers were selected and 95 of these were entirely analyzed. As a result, we identified eleven factors of influence on sustainability practices in the consumer behavior of sustainable products (environment, age, educational level, economic class, gender, conscience, self-image concern, emotion, legislation, access to information, trust and habit) and seven about consumer attributes and perception about sustainability (eco-friendly, origin assurance, consumer health, fair trade, animal testing, recyclables, and natural ingredients).

Keywords: sustainable consumption; consumer behavior; sustainability; attribute

Recebido em: 05/12/2018 Revisado em: 02/04/2019 Aprovado em: 26/09/2019

Check for updates

\section{Luciana Faluba \\ Damázio (iD,}

Fundação Dom Cabral, Brasil Doutora em Administração pela Universidade Federal de Minas Gerais, Brasil

luciana.faluba@fdc.org.br

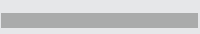

Leonardo Antunes

Nogueira Coutinho (iD,

Universidade Federal de Minas Gerais, Brasil

Graduado em Administração pela Universidade Federal de Minas Gerais, Brasil

leonardoanc@hotmail.com

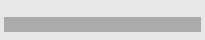

Helena Belintani Shigaki iD,

Centro Universitário Unihorizontes, Brasil

Doutora em Administração pela Universidade Federal de Minas Gerais, Brasil

belintanihs@gmail.com 


\section{Introdução}

O consumo sustentável tem recebido destaque desde a elaboração da Agenda 21, um conjunto de resoluções produzido pela Conferência Internacional Eco-92, realizada no Rio de Janeiro em 1992. Pouco antes, na década de 1980, já havia uma preocupação do impacto do consumo no meio ambiente (Ribeiro, Veiga, \& Higuchi, 2016), o que ajudou a consolidar o termo consumo verde - considerado uma ramificação do consumo sustentável. O consumo de produtos ecológicos ainda está em seu início e corresponde a no máximo $5 \%$ do consumo mundial total há alguns anos. Entretanto, o número está em ascensão, tendo em vista que a cada dia a sociedade tem se declarado sensibilizada com a questão da sustentabilidade (Lipovetsky, 2006).

No Brasil, o Instituto Brasileiro de Geografia e Estatística (IBGE) publica, desde 2002, os indicadores de desenvolvimento sustentável, como esforço "para concretização das ideias e princípios formulados na Conferência das Nações Unidas sobre Meio Ambiente e Desenvolvimento" (p. 1), com enfoque para as dimensões ambiental, social, institucional e econômica. Balderjahn et al. (2013) desenvolveram uma escala que mensura o nível de consciência em consumo sustentável, mensurando-o frente às questões de meio ambiente, social e econômico. Já um estudo realizado pelo Massachusetts Institute of Technology (MIT) indica que há um crescente número de investidores preocupados com o desempenho ambiental e social dos negócios, além do desempenho financeiro (Unruh et al., 2016).

A pesquisa por novas teorias e práticas do comportamento do consumidor aparece não só como uma forma de revelar novos padrões de consumo, mas também como forma de criar novas oportunidades de negócios (Schafer, Jaeger-Erben, \& Santos, 2011). E, como alertam Nespolo et al. (2016), "assiste-se à emergência de um novo tipo de postura dos indivíduos, a qual pondera os impactos de seu padrão de consumo na natureza" (p. 138).

Nesse sentido, à título de problematização, Lipovetsky (2006) fala da "civilização do desejo", que "preconiza o incitamento perpétuo da procura, da comercialização e da multiplicação indefinida das necessidades: o capitalismo de consumo assumiu a liderança das economias de produção" (p. 7). Em momentos de intensas ameaças de catástrofes ecológicas, observa-se uma preocupação com o hiperconsumismo, que surge como uma prática a ser responsabilizada com urgência, uma vez que o consumo excessivo pode desequilibrar a ecosfera. O hiperconsumidor existe, portanto, diante da sua constante busca pela experiência emocional, bem-estar, qualidade de vida, saúde, autenticidade, imediatismo e pela comunicação, que reforçam a necessidade de consumir (Lipovetsky, 2006).

As pesquisas sobre o tema aqui estudado ainda são dispersas e incipientes, apesar de apresentarem um crescimento significativo nos últimos cinco anos, que representou um total de $75 \%$ das publicações aqui selecionadas. Nesse sentido, faz-se relevante uma ampla análise das publicações acadêmica e profissionais, com vistas a compreender o que já foi produzido e indicar caminhos para pesquisas futuras. 
Diante do exposto, a partir dessa revisão sistemática de literatura, tem-se a seguinte questão norteadora: qual o estado da arte do conhecimento sobre consumo de produtos sustentáveis? Para tanto, o presente artigo tem por objetivo geral realizar uma revisão sistemática de literatura sobre o comportamento do consumidor em relação aos produtos sustentáveis e os seguintes objetivos específicos, respectivamente: (a) identificar os fatores que interferem no comportamento e (b) definir os atributos de sustentabilidade e a percepção do consumidor em relação à sustentabilidade.

Esse panorama aqui apresentado revela a importância de se estudar e identificar os diferentes aspectos relacionados ao consumo sustentável, visto a diversidade de estudos e dimensões utilizadas em nível governamental, institucional e acadêmico. Ao apresentar, como resultado final, os onze fatores de influência nas práticas de sustentabilidade no comportamento do consumidor de produtos sustentáveis e os sete em relação aos atributos e percepção do consumidor acerca de sustentabilidade, os autores contribuem com a literatura na área, que ainda é escassa e inconclusiva apesar de sua notória importância para o meio ambiente a curto e longo prazo.

Por justificativa, entende-se que, comparado há alguns anos, a população tem desenvolvido uma consciência dos problemas ambientais que o mundo vem enfrentando, tais como poluição, desmatamento, aquecimento global, entre outros. E, ao repensarem suas atitudes, a fim de adotarem práticas mais sustentáveis, os consumidores passam a exigir um comportamento socialmente responsável dos dirigentes das empresas (Ribeiro, Veiga, \& Higuchi, 2016). Cada vez mais há uma tendência em adquirir novos hábitos de consumo, dando preferência a produtos sustentáveis - recicláveis e mais duráveis, mesmo que esses tenham um valor mais elevado que aqueles que não o são (Leonidou, Katsikeas, \& Morgan, 2013; Guillen-Royo, 2019).

No entanto, infelizmente, observa-se ainda que grande parcela da população permanece com hábitos pouco sustentáveis e ainda não está sensibilizada para essa questão, apesar da consciência de seus efeitos na sociedade. Uma das razões desse acontecimento é o fator motivacional (Hüttel, Ziesemer, Peyer, \& Balderjahn, 2018) em que os consumidores evitam resultados negativos para instantes de alegria e bem-estar. Portanto, a principal contribuição do estudo envolve uma análise ampla sobre o conhecimento acerca do tema. E, para isso, a presente pesquisa configura-se como uma revisão sistemática de literatura frente ao comportamento do consumidor em relação aos produtos sustentáveis.

\section{Fundamentação Teórica}

O tema proposto neste trabalho foi estudado de forma dinâmica e inter-relacionada, tendo como parâmetro as teorias que abarcam os temas de comportamento do consumidor e produtos sustentáveis.

Em resumo, o campo do comportamento do consumidor abrange uma ampla área de estudo e pode ser considerado como a busca pelo entendimento do comportamento de indivíduos ou grupos quanto aos processos de seleção, compra, uso e descarte de produtos, serviços, ideias ou experiências para satisfazer suas necessidades e desejos (Solomon, 2008). Silva (2012) 
corrobora afirmando que "o consumir reflete as práticas individuais, nas quais cada um possui a condição de escolha sobre suas ações" (p. 223).

Os clientes formam seus julgamentos sobre um determinado produto, serviço ou experiência a partir de múltiplas variáveis - cultura, classe social, experiências adquiridas, grupos de referência, aprendizagem, emoção, memória, entre outros (Espinoza, 2004). Ultimamente os estudos sobre o comportamento do consumidor têm extrapolado os modelos integrativos já consagrados de tomada de decisão (vide autores como Howard-Sheth (1969); Nicosia (1966); Engel, Blackwell e Miniard (1986)), para também dar atenção aos estudos sobre emoção, memória sensibilização e engajamento, por exemplo.

Além do mais, a Internet, mídias sociais e os dispositivos móveis estão revolucionando as indústrias de bens de consumo, as empresas e também o comportamento dos consumidores. A articulação dos novos meios de comunicação e a descoberta e exploração de novos canais possibilitam à empresa alcançar e conectar os clientes com a própria empresa ou mesmo com outros clientes. A partir dessa interação, surgem formatos de relacionamento que permitem que um número crescente de clientes participe de forma ativa na criação de conteúdo sobre os produtos e experiências de compra (cocriação de valor) e criação e manutenção de comunidades de marcas (Grönroos \& Voima, 2013; Vargo \& Lusch, 2016). A confiança, considerada um fator importante na tomada de decisão, corrobora com a estruturação da proposta de valor e a articulação da comercialização da organização. Mais informados, os consumidores esperarão por mais transparência e honestidade das empresas (Damázio, Gonçalves, \& Shigaki, 2017).

Acreditando que cada vez mais os consumidores estarão preocupados com o desenvolvimento sustentável, deve-se pensar no consumo sem a necessidade de agressão ao meio ambiente e à sociedade (Funaru \& Baranov, 2012), respeitando a relação entre bem-estar e consumo sustentável no âmbito hedônico - da alegria, cognitivo - da satisfação, e no subjetivo (Guillen-Royo, 2019). Esse é o desafio. A definição de desenvolvimento sustentável se relaciona com o conceito de satisfação das necessidades das gerações atuais sem prejudicar as próximas.

O consumo sustentável é aquele no qual há preferência, por parte do consumidor, por produtos ecológicos, naturais, recicláveis, que não prejudiquem sua saúde ou mesmo o meio ambiente (Joshi \& Rahman, 2015).

As empresas, por exemplo, podem encorajar clientes a adotarem comportamentos que preservem o meio ambiente e a eliminar os que o prejudicam, atuando na linha de frente em prol da sustentabilidade (Rettie, Burche, \& Riley, 2012), com a criação de produtos sustentáveis. Além disso, os consumidores esperam ter participação no desenvolvimento de novos produtos e serviços, em particular nas empresas que eles se sentem leais. Esse desenvolvimento deve ser acompanhado pelos pilares da responsabilidade social, transparência e sustentabilidade.

Ademais, é aconselhável elaborar um modelo de negócios com proposições, criação e captura de valores entregues aos stakeholders, conforme sugerido por Tunn, Bocken, Hende e Schoormans (2019), em que os autores identificaram que esses fatores, que envolvem desde o 
desenvolvimento do produto e serviço até a sua estratégia de crescimento sustentável, auxiliam na redução de diferentes níveis de consumo e também no esforço do consumidor no processo de tomada de decisão. Essa afirmação corrobora com o estudo de Guillen-Royo (2019) quanto a discussão de consumo sustentável e bem-estar do consumidor.

A sustentabilidade "engloba uma série de direcionamentos corporativos que impactam profundamente a dinâmica de atuação da empresa e o modelo de trabalho de seus colaboradores diretos e indiretos, bem como seu impacto nos diversos stakeholders" (Strategy Partners, 2010, p. 1), corroborando com o processo de criação de valor da organização. De acordo com Luchs, Naylor, Irwin e Raghunathan (2010), produtos sustentáveis são aqueles que possuem bons atributos sociais ou ambientais. Esse conceito foi o que se mostrou mais consistente com o trabalho desenvolvido nesse artigo.

\section{Procedimentos Metodológicos}

O método de Revisão Sistemática de Literatura se revelou eficiente e criterioso para identificação e avaliação de extensas literaturas (Mulrow, 1994). Tal distinção se dá, principalmente, pela imparcialidade do método em comparação às revisões tradicionais (Tranfield, Denyer, \& Smart, 2003), por apresentar um protocolo rígido e explícito para identificar, selecionar, coletar e analisar as contribuições relevantes à pesquisa (Sampaio \& Mancini, 2007). A Figura 1, adaptada pelos autores deste artigo a partir de Holmes (2010), indica as etapas que foram realizadas para este estudo.

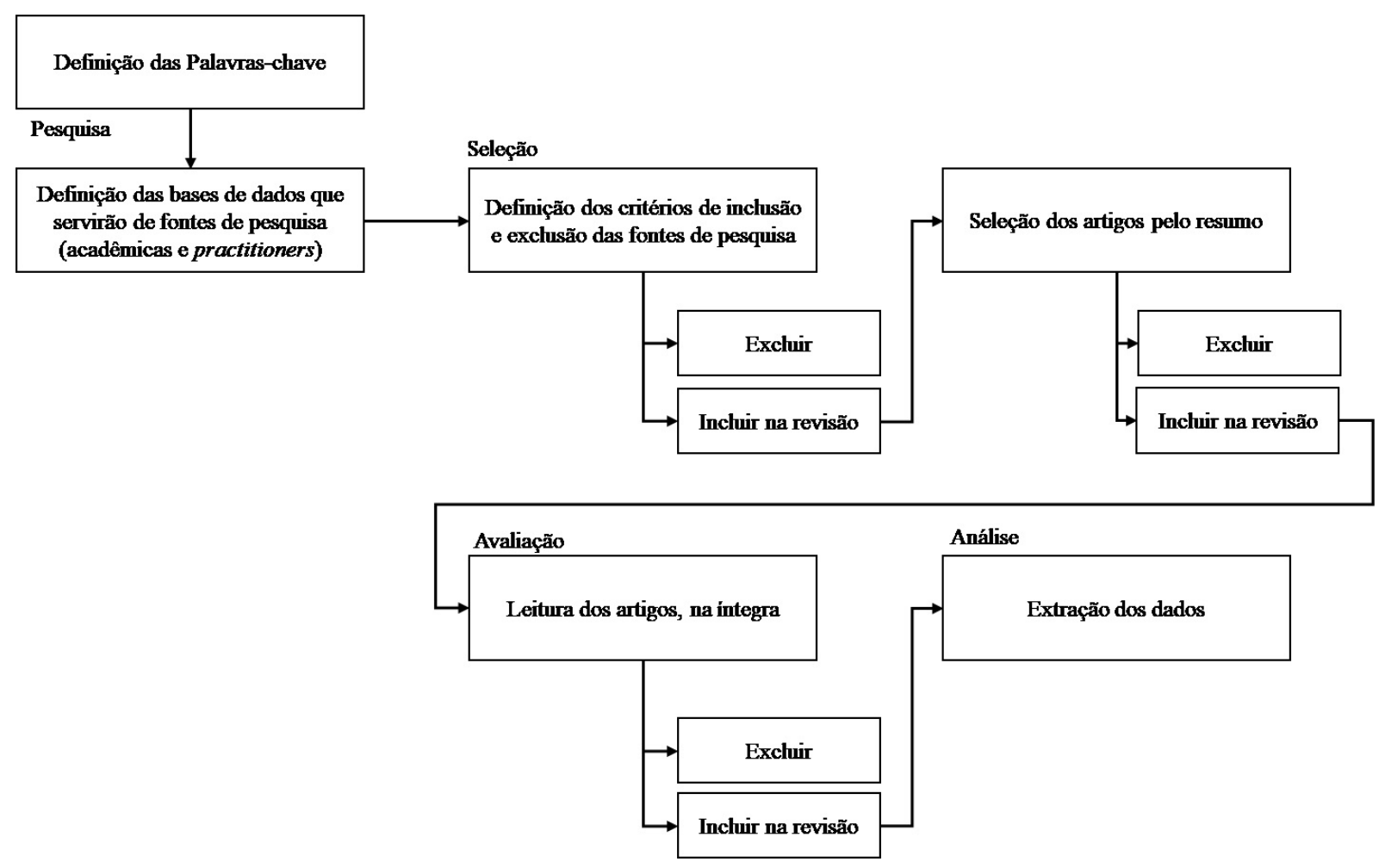

Figura 1. Visão geral do método revisão sistemática de literatura.

Fonte: Adaptado pelos autores a partir de Holmes (2010). 
A partir da definição do escopo da pesquisa, este estudo iniciou-se com a identificação de palavras-chave e termos de busca (Tranfield, Denyer, \& Smart, 2003). As palavras-chave de cada um dos três tópicos encontram-se na Figura 2. Danciu (2013) alertou que com o aumento da população mundial, há também o crescimento do consumo de produtos sustentáveis em países em desenvolvimento e emergentes, devido ao aumento significativo da classe média nesses mercados. Por isso, houve a inclusão dos termos "Classe Média", "Países em Desenvolvimento" e "Brasil", para, além do intuito desta pesquisa, dar uma atenção especial a esses mercados.

\begin{tabular}{ll}
\hline \multicolumn{1}{c}{ Tópicos } & \multicolumn{1}{c}{ Palavras-Chave } \\
\hline & Consum* \\
Atributos Sustentáveis dos Produtos e sua & Sustainab* $^{*}$ \\
relação com o consumo. & $\begin{array}{l}\text { Attributes OR Features OR Benefits } \\
\text { Behaviour OR Behavior OR Journey }\end{array}$ \\
\hline & Consum \\
Consumo de Produtos Sustentáveis da Classe & Sustainab* \\
Média no Brasil. & Class C OR Middle Class \\
& Brazil \\
\hline & Consum \\
Consumo de Produtos Sustentáveis da Classe & Sustainab* \\
Média em Países em Desenvolvimento. & Class C OR Middle Class \\
& Developing Countries \\
\hline
\end{tabular}

Figura 2. Palavras-chave da revisão sistemática

Fonte: Elaborado pelos autores

Além da delimitação de termos de busca ou palavras-chaves, na fase inicial do estudo é importante determinar quais serão as bases de dados, ou fontes de pesquisa, analisadas. Com o objetivo de ampliar o escopo desse estudo e dada a natureza recente dessa temática, a pesquisa foi conduzida não só em fontes acadêmicas e bases de dados listadas, mas também em bases practitioners (profissionais).

A pesquisa foi realizada em 2019 e foram identificados artigos publicados de 2007 (ano em que o primeiro estudo com foco no escopo deste artigo foi publicado) a 2018. Dentre as bases acadêmicas, foram utilizadas: EBSCO, Google, Portal Capes de Periódicos e Google Scholar. Quanto as bases practitioners, foram utilizadas: SustainAbility, AccountAbility, KPMG, Boston Consulting Group (BCG), McKinsey, World Business Council for Sustainable Development, Mackenzie Brasil, Instituto Akatu, Darmouth, CEBDS, Greenbiz, PwC, Deloitte, Instituto Ethos, Accenture, Sustainability, OECD e, The Economist.

Os critérios para inclusão e exclusão (Figura 3) foram documentados (Tranfield, Denyer, \& Smart, 2003), o que torna o processo de pesquisa explícito e transparente, assegurando o foco da pesquisa nas áreas importantes de estudo e não em conhecimentos marginais (Holmes, 2010). O número de fontes incluídas e excluídas foram registradas em cada estágio da revisão (Tranfield, Denyer, \& Smart, 2003). 


\begin{tabular}{ll}
\hline \multicolumn{1}{c}{ Critério de Inclusão/Exclusão de Literatura } & Motivo \\
\hline Textos que não se propunham a analisar o Consumo foram excluídos & A pesquisa se delimita a analisar os hábitos de consumo \\
\hline Textos de estudos de regiões de países desenvolvidos foram incluídos & $\begin{array}{l}\text { A teoria desenvolvida nessas regiões corrobora com os estudos } \\
\text { de regiões brasileiras }\end{array}$ \\
\hline Textos que não analisam aspectos sustentáveis foram excluídos & 0 escopo da pesquisa se restringe à sustentabilidade \\
\hline
\end{tabular}

Figura 3. Critérios de inclusão/exclusão

Fonte: Elaborado pelos autores

Foram identificados mais de 4600 textos e, usando os critérios de inclusão e exclusão ora estabelecidos, foram selecionados 219 artigos a partir da leitura dos resumos. Ao final, 95 textos foram analisados, sendo 83 artigos acadêmicos e 12 practitioners.

Os conceitos e as considerações específicas sobre o assunto foram analisados a partir da técnica de análise de conteúdo, cujo tema torna-se uma unidade de significação que se liberta do texto analisado e pode ser traduzido por um resumo, por uma frase ou por uma palavra (Bardin, 1979). Os artigos analisados passaram pelos seguintes passos analíticos: (a) identificação das ideias centrais dos trechos transcritos de todos os artigos; (b) classificação dos sentidos subjacentes às ideias em temas que resumem a produção do conhecimento acerca do assunto estudado e (c) elaboração de sínteses interpretativas de cada tema.

\section{Apresentação e Análise dos Dados}

Percebe-se, na literatura, que o interesse acerca do tema abordado nessa pesquisa vem crescendo, uma vez que todos os artigos selecionados foram publicados entre 2007 (ano em que o primeiro estudo, com foco no escopo deste artigo, foi publicado) e 2018, com crescimento significativo nos últimos cinco anos, que representou um total de $75 \%$ das publicações selecionadas (Figura 4).

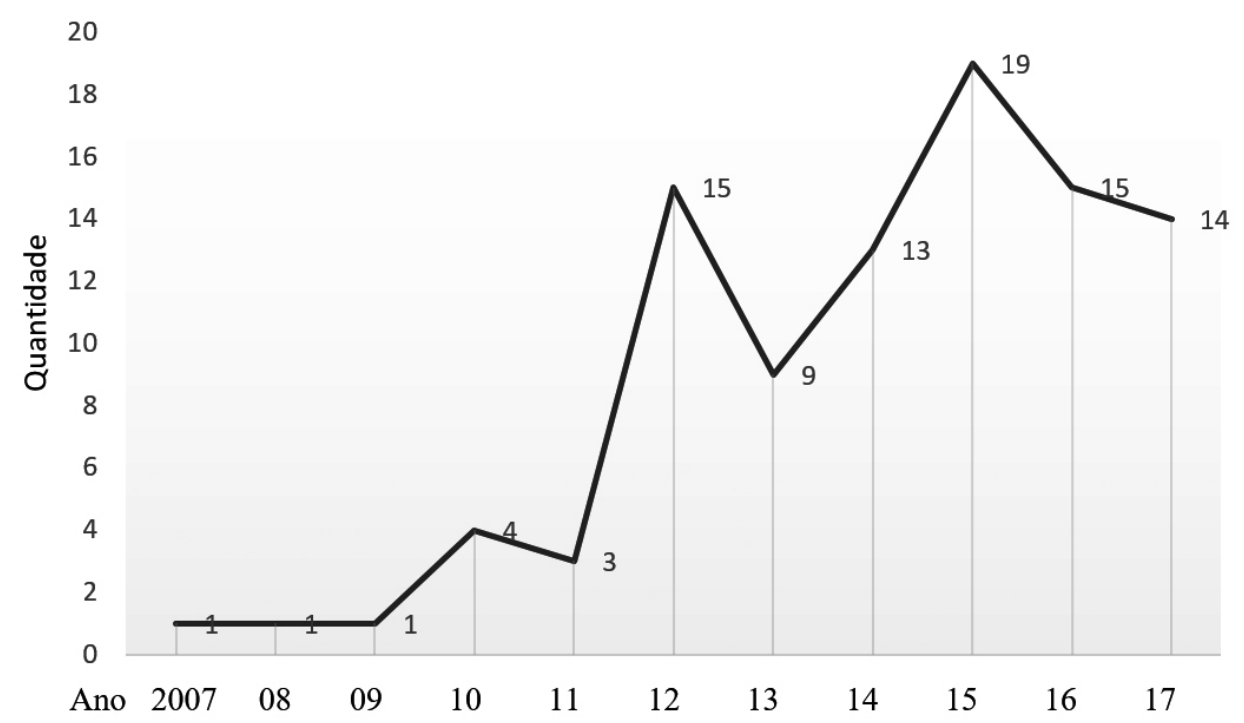

Figura 4. Quantidade de publicação por ano

Fonte: Elaborado pelos autores 
Com relação aos métodos de pesquisa utilizados, predominam os artigos com abordagem quantitativa (52 artigos), seguido por artigos teóricos (17), com abordagem qualitativa (12), métodos mistos (7), observação (4) e estudo de caso (3). Do mesmo modo, pode-se afirmar que menos de 5\% dos artigos practitioner analisados contribuíram significativamente com os resultados encontrados. Ou seja, todos os resultados aqui indicados vieram de artigos acadêmicos. Nas próximas subseções foram discutidos os fatores que interferem no comportamento do consumidor em prol da sustentabilidade e os atributos de sustentabilidade e a percepção dos consumidores que enquadram um produto como sustentável em relação a esses atributos.

\section{Fatores que influenciam o consumo de produtos sustentáveis}

Foram identificados, neste estudo, 11 fatores de influência nas práticas de sustentabilidade no comportamento do consumidor de produtos sustentáveis, a saber: ambiente, idade, nível educacional, classe econômica, gênero, consciência, preocupação com a própria imagem, emoção, acesso à informação, confiança do consumidor e hábito (Figura 5).

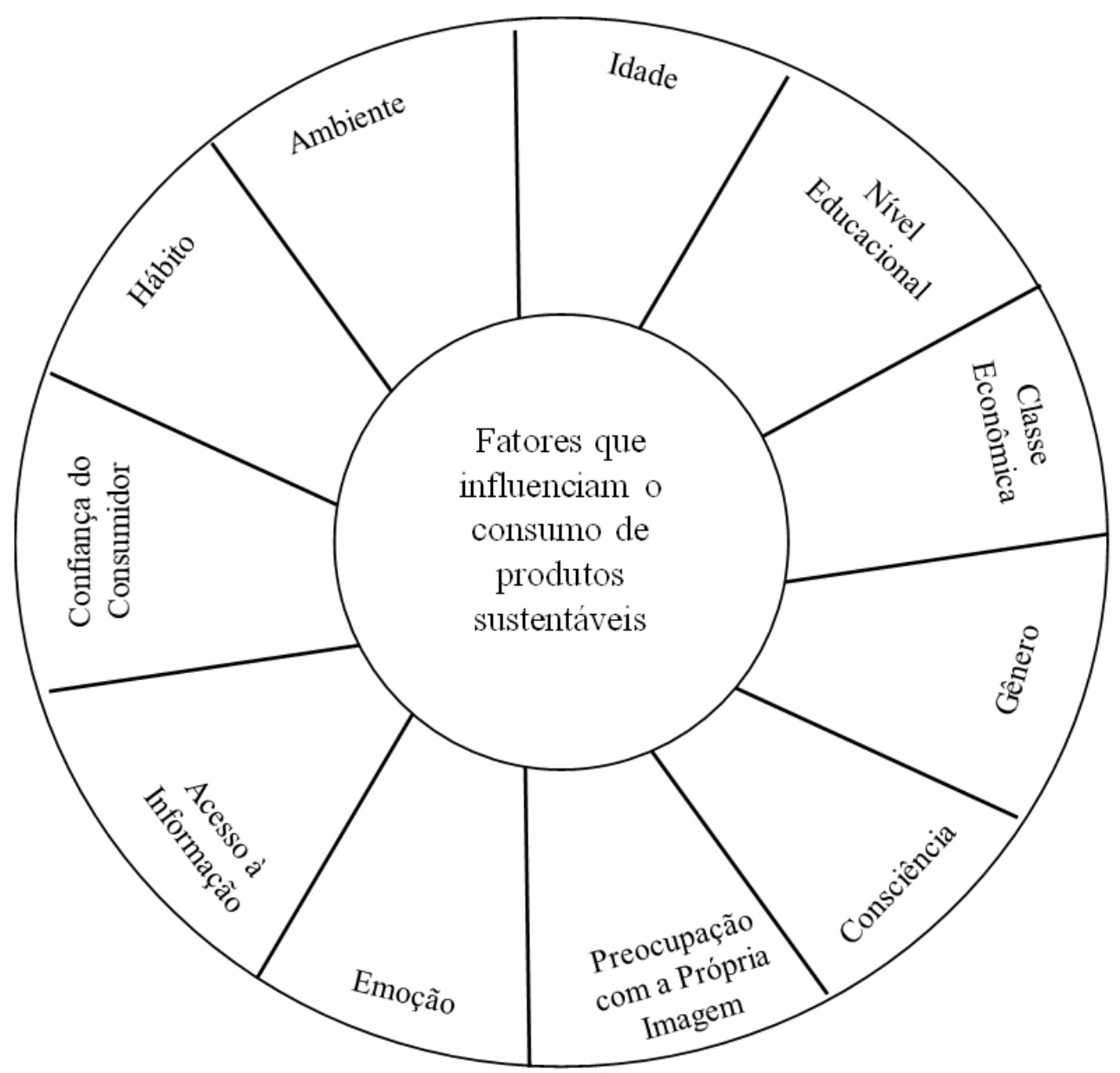

Figura 5. Fatores que influenciam o consumo de produtos sustentáveis

Fonte: Elaborado pelos autores 
O ambiente se dá tanto de forma macro quanto micro. Instituições como a sociedade, a comunidade e a família têm seus conjuntos de normas, crenças, valores e hábitos e, desse modo, um papel influenciador no comportamento do indivíduo. A percepção de valor passa a ser algo cultural e inerente ao indivíduo, interferindo no ato de compra (Monteiro, Giuliani, Zambon, Pizzinatto, \& Cunha, 2012; Hosseinpour, Nezakati, Sidin, \& Fee, 2016). Além dos já citados, no fator ambiente estão presentes categorias como fatores situacionais, disponibilidade do produto e imagem da marca (Joshi \& Rahman, 2015) e legislação.

A insuficiência legal em alguns países, em relação ao meio ambiente, é um problema de alta relevância no quesito desenvolvimento sustentável nacional. Apesar do grande esforço para a mudança do quadro legislativo, as empresas também podem se mostrar como grandes influenciadoras do caminho para um sistema legal mais sustentável. Estudos revelam que ambientes com legislação ambiental mais rigorosa influenciam em provocar práticas mais sustentáveis nos indivíduos (Kalantari \& Asadi, 2010 apud Carrete, Castaño, Felix, Centeno, \& González, 2012). Esse fator, embora relevante, não tem sido discutido com a mesma ênfase que os demais na academia. Consumidores mais jovens tendem a ser aqueles que apresentam práticas voltadas para a sustentabilidade, com comportamentos de maior consciência socioambiental (Carrete et al., 2012). A idade influencia na consciência ambiental de um grupo e na esperança para o futuro, evidenciando que as novas gerações tendem a ter maior preocupação socioambiental, bem como aqueles que não enfatizam o consumo, indicando comportamentos de frugalidade (Ronchi, Oliveira, Parisotto, \& Gomes, 2016). Já o nível educacional tem influência no consumo consciente, à medida que pessoas com níveis mais altos de escolaridade tendem a possuir maior consciência e responsabilidade em seus comportamentos ambientais (Schäufele \& Hamm, 2015).

A classe econômica é uma variável relevante, uma vez que pessoas com maior poder aquisitivo têm também maior autonomia para arcar com os custos adicionais dos produtos que observem a sustentabilidade. Ademais, as classes mais pobres tendem a fazer maior reuso dos produtos comprados e reciclam mais, diante da economia que se atinge (Schafer, Jaeger-Erben, \& Santos, 2011; Carrete et al., 2012; Schäufele \& Hamm, 2015).

O gênero, por sua vez, foi estudado por Schäufele e Hamm (2017) ao incluí-lo como antecedente no modelo de comportamento de compra de vinhos com características de sustentabilidade. Partiu-se do pressuposto de estudos anteriores que o homem estaria disposto a pagar mais pelo vinho. Em contrapartida, Ramirez, Jiménez e Gau (2015) e Stranieri, Ricci e Banterle (2017) afirmam que a mulher apresenta maior predisposição a comprar produtos sustentáveis quando comparado aos homens.

Apesar de muitas vezes haver um distanciamento entre consciência e atitude, é inegável que a consciência tem forte influência sobre as práticas sustentáveis. Indivíduos que apresentam maior apelo à causa sustentável (Monteiro et al., 2012; Ramirez, Jiménez, \& Gau, 2015; Rothenberg \& Matthews, 2017) e que praticam valores como autorrespeito, autorrealização e relacionamento caloroso com seus próximos, estão mais próximos do comportamento sustentável (Simões, Giraldi, \& Oliveira, 2012). 
Porém, estes mesmos indivíduos, quando estão sendo observados por outros, tendem a optar por benefícios coletivos a despeito dos individuais (Green \& Peloza, 2014; Ramirez, Jiménez, \& Gau, 2015). A preocupação com a própria imagem revelou-se um fator instigante, pois ao mesmo tempo em que há uma preocupação de autoconceito em satisfazer suas necessidades pessoais frente a aprovação da sociedade, observa-se também que há benefícios sociais neste comportamento no momento em que "ao ser visto como alguém que cuida da comunidade e do meio ambiente, um consumidor pode garantir que ele/ela atenda padrões sociais e padrões comunitários que reforcem tais comportamentos" (Kataria, Mukherjee, Biswas, \& Garg, 2016, p. 58). Associado a esse fator e às preocupações ambientais, tem-se as emoções que, se possuírem intensidade e valência positiva em relação ao aspecto sustentável, há um impacto direto na intenção de compra de produtos nesta categoria (Joshi \& Rahman, 2015).

O acesso à informação atua como fator determinante no molde desses padrões de comportamento no ambiente, como a exposição à comunicação de massa ou a ambientes selecionados - escolas, varejo e empresas, por exemplo - por diferentes canais. Anúncios que destacam o compromisso de uma empresa em relação à sua preocupação frente aos impactos sociais e ambientais podem influenciar a compra de 34\% dos consumidores (Nielsen, 2015).

A confiança do consumidor está relacionada à construção de um relacionamento confiáveljuntoaofornecedor, sendomedidapelatransparência, reputação e lembrança de ações passadas da empresa (Schäufele \& Hamm, 2017). Segundo os autores, esse fator pode ser considerado como um dos mais importantes para a prática de comportamentos socioambientais, já que pode influenciar a formação de consciência no indivíduo, sua preocupação com a própria imagem e também o hábito.

Por fim, o estudo de Stranieri, Ricci e Banterle (2017) indica que a intenção de compra pode ser um preditor para a formação de hábito no comportamento do consumidor, que pode ser reforçado por associações positivas e levar à repetição do ato. Consequentemente, se há entrega de valor ao consumidor, sua experiência com a marca ou um fenômeno será positiva. Em um estudo bibliométrico realizado por Joshi e Rahman (2015), os autores elucidaram que a loja física também pode vir a ser um fator de influência na compra de produtos sustentáveis, apesar desse tema carecer de estudos.

\section{Atributos Sustentáveis e percepção do consumidor}

Como resultado da Revisão Sistemática, foram aqui descritos os atributos de sustentabilidade dos produtos e seu impacto na percepção do consumidor. Assim sendo, os achados foram: Eco-friendly, garantia de origem, saúde do consumidor, fair trade, teste em animais, recicláveis e ingredientes naturais (Figura 6). 


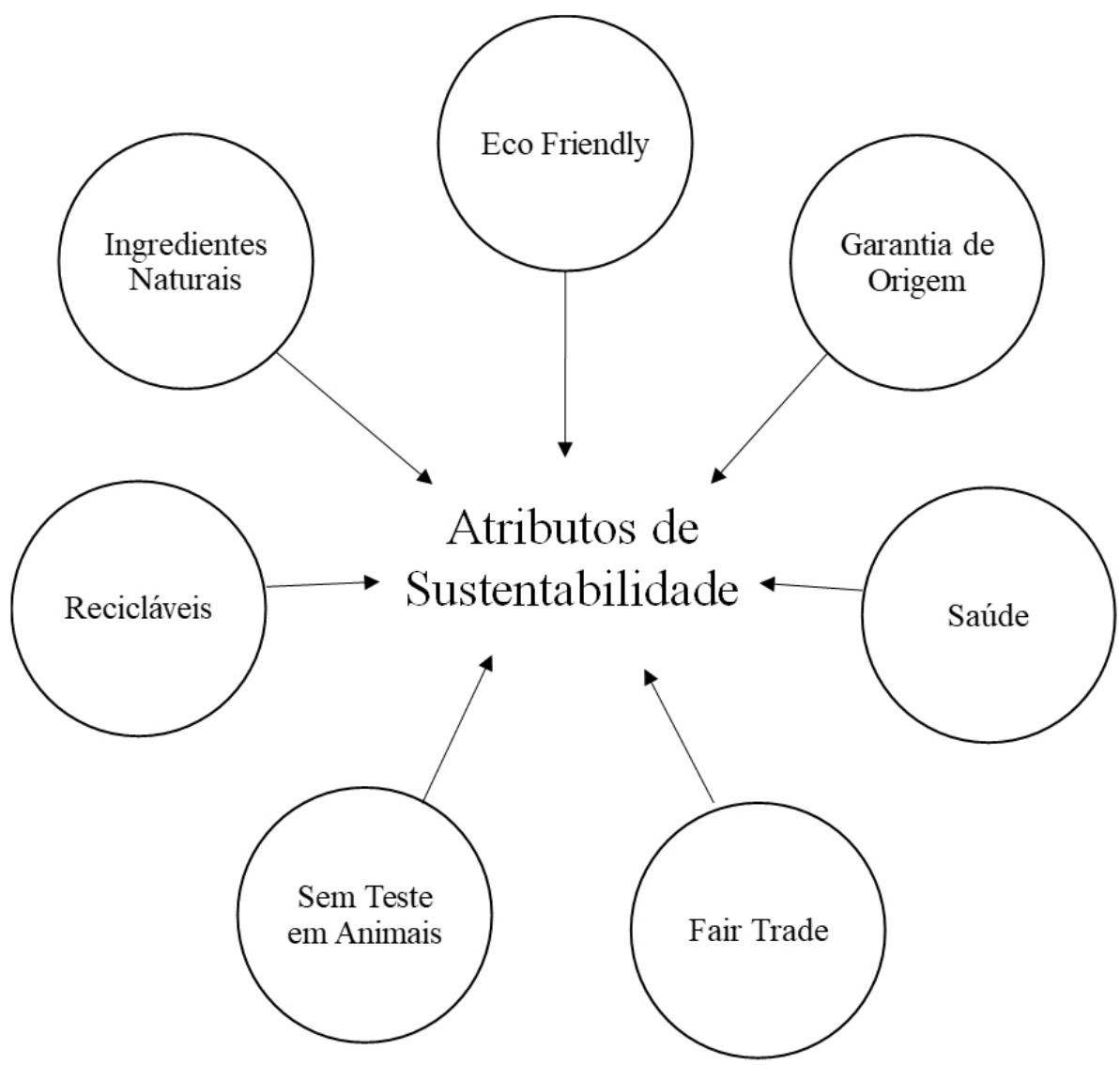

Figura 6. Atributos sustentáveis e percepção do consumidor

Fonte: Elaborado pelos autores

Pesquisas realizadas por Hoek, Roling e Holdsworth (2013) mostraram que no setor alimentício, o termo Eco-Friendly é tido como um produto composto por ingredientes de maior qualidade e que o produto é mais saudável por não possuir corantes e aromatizantes ou outras substâncias artificias. Ainda segundo os autores, o termo eco-friendly é compreendido como algo de menor dano ao meio ambiente e melhores condições de manufatura. Essa mesma pesquisa mostrou que o termo é muitas vezes visto como um "clichê" vago.

Adicionalmente, Biswas (2016) afirma que o baixo impacto no meio ambiente, advindo da produção e consumo dos produtos eco-friendly, tem sido um fator relevante para o consumidor. Em contrapartida, Kostadinova (2016) assegura que produtos eco-friendly são mais caros e normalmente de baixa qualidade, quando comparados à grande quantidade de produtos não sustentáveis disponíveis no mercado. O grupo de consumidores dessa categoria pode ser chamado de LOHAS (Lifestyles of Health and Sustainability) que, segundo Ottman (2012), são aqueles mais conscientes e ativos quanto ao ambiente, percebendo uma "ligação universal entre saúde e preservação do meio ambiente" (p. 56). Mas, ao mesmo tempo, podem ser considerados drifters, indivíduos motivados por tendências que chegam a ultrapassar seus ideais, ou seja, consideram este estilo de vida uma modinha (Ottman, 2012). 
A garantia de origem é evidenciada pela informação da origem do produto e também se a produção é local ou não. Produtos de origem em países em desenvolvimento remetem os consumidores a práticas insustentáveis, enquanto em países desenvolvidos geram impressão de cuidado socioambiental (Gruber, Schlegelmilch, \& Houston, 2014; Rothenberg \& Matthews, 2017). É o caso, por exemplo, da Original Unverpackt (original, sem embalagem), uma empresa Alemã inaugurada em 2014 em Berlin, que trabalha com a venda de produtos sem embalagem, com compras a granel em que os próprios clientes levam as embalagens que possuem em casa ou, se preferirem, podem adquirir saquinhos de papel biodegradável. A empresa é hoje considerada um sucesso e tem conquistado prêmios de melhores ideias de marketing e negócios sustentável.

No setor alimentício, produtos com selos de garantia de origem são considerados aqueles mais seguros, com mais qualidade e maior respeito ao meio ambiente (Gruber, Schlegelmilch, \& Houston, 2014). Nesse mesmo setor, 34\% das pessoas classificaram as certificações de Garantia de Origem como grau 4 ou 5 de importância no ato da compra, numa escala de 5 pontos (Annunziata \& Scarpato, 2014), indicando qualidade (Megicks, Memery, \& Angell, 2012), condições justas de trabalho, menor emissão de carbono e apoio à economia nacional e local (Hoek, Roling, \& Holdsworth, 2013).

Os atributos ligados à saúde do consumidor têm sido uma preocupação crescente devido aos diversos escândalos de contaminação e adulteração em alimentos (Cunha, Spers, \& Zylbersztajn, 2011). No estudo de Annunziata e Scarpato (2014), esse foi o segundo atributo mais importante na compra de alimentos, em que $70 \%$ das pessoas classificaram essas certificações como grau 4 ou 5 de importância no ato da compra, em uma escala de 5 pontos. O grupo de consumidores predisposto a ser influenciado por esse fator pode ser chamado de naturalites, que segundo Ottman (2012) são aqueles que adotam um estilo de vida saudável e se preocupam com os efeitos prejudiciais dos produtos químicos nos produtos que consomem. Os consumidores procuram, portanto, por produtos que sejam bons para ele e para a sociedade, ocasionando bem-estar do início ao fim de uma ação (Nielsen, 2015).

Pesquisas feitas por Barbosa e Veloso (2012) revelam que apenas 1\% dos brasileiros afirmam conhecer bem o significado de Fair Trade. Exatamente por isso, produtos com esse atributo parecem ter pouco impacto sobre o consumo (Annunziata \& Scarpato, 2014; Hoek, Roling, \& Holdsworth, 2013). Fair Trade, ou comércio justo, em resumo, é a prática legal da empresa oferecer melhores condições de trabalho aos seus funcionários. De acordo com o estudo de Annunziata e Scarpato (2014), $48 \%$ dos que consumem produtos Fair Trade o fazem porque consideram ético, $42 \%$ por respeito aos empregados e somente $5 \%$ por motivos pessoais. O estudo de lqbal (2011) indicou que a disposição em pagar mais pelo atributo Fair Trade no setor de vestuário é estatisticamente irrelevante. São discutidos, ainda, temas relacionados aos direitos humanos e boas práticas com funcionários. 
A maioria dos entrevistados do artigo de Hoek, Roling e Holdsworth (2013) afirmaram ter pouco conhecimento sobre o que é permitido em relação a testes em animais e sobre a ocorrência destes. Alguns conseguiram associar o atributo de cuidados aos animais a algumas marcas e outros consideraram uma estratégia de marketing que pode não condizer com a realidade. Pela pesquisa de Hammerle (2012), os respondentes se mostraram dispostos a pagar em média 22\% a mais por esse atributo no setor de produtos orgânicos, sendo esse o que demonstra maior impacto na intenção de compra do consumidor.

Em pesquisa realizada por Hoek, Roling e Holdsworth (2013), os participantes criticaram o termo reciclável por sua ambiguidade. Alguns entendiam que o termo se referia a uma embalagem feita com produtos reciclados, que a embalagem poderia ser reciclada, ou que o termo encoraja o consumidor a reciclar. O grupo de consumidores mais afeito ao consumo desses produtos é o conventionals (Ottman, 2012), ou seja, aqueles que têm consciência das questões ambientais, reciclam e reutilizam produtos na tentativa de economizar dinheiro. Em um estudo realizado por Ronchi et al. (2016), apesar dos participantes da pesquisa terem acesso à coleta seletiva, esse foi o determinante menos evidente na pesquisa que poderia influenciar em um comportamento mais sustentável.

$O$ fator ingrediente natural pode ser entendido como um produto orgânico ou livre de modificações genéticas e químicas. Pelas pesquisas desenvolvidas por Barbosa e Veloso (2012), 24\% dos brasileiros entrevistados conhecem bem, ou muito bem, o termo orgânico. Na Itália, por exemplo, $38 \%$ dos respondentes conheciam o termo (Annunziata \& Scarpato, 2014). Annunziata e Scarpato (2014) revelam também que 30\% dos respondentes da pesquisa compram esse tipo de produto com frequência e 20\% ocasionalmente. Essa frequência de consumo também se reflete nas pesquisas, em que o objeto de estudo mais recorrente nos artigos dessa revisão sistemática da literatura foram os produtos orgânicos (Hammerle, 2012; Hamzaoui, \& Zahaf, 2009; Holmes, 2010).

Quanto ao motivo da compra, $42 \%$ compram o produto com ingrediente natural por motivo de saúde, 36\% pelo ambiente e 12\% por prazer (Hamzaoui \& Zahaf, 2009). Novos processos, até então de difícil compreensão, como a irradiação e a transgenia de alimentos, reforçam as dúvidas e os riscos percebidos pelo consumidor de alimentos modificados geneticamente (Cunha, Spers, \& Zylbersztajn, 2011). 65\% das pessoas classificam as certificações "produto não geneticamente modificado" como grau 4 ou 5 de importância no ato da compra, numa escala de 5 pontos (Annunziata \& Scarpato, 2014).

Outros atributos também foram encontrados neste estudo, porém sem impacto relevante na percepção do consumidor: produto reutilizável, livre de emissões de carbono, agricultura sustentável, madeira sustentável, embalagem com cuidado ambiental e eco eficiente (Produto com baixa utilização de água ou energia). 


\section{Conclusões e Recomendações}

A partir desse estudo, foi possível identificar os fatores que interferem no comportamento do consumidor em prol da sustentabilidade e identificar os atributos de sustentabilidade que enquadram um produto como sustentável, e a apresentação dos estudos de percepção do consumidor em relação a esses atributos. Desse modo, a pesquisa contribui para a literatura vigente, ao ser categórica em suas definições e servir de referencial teórico para pesquisas futuras, com a identificação de 11 fatores: ambiente, idade, nível educacional, classe econômica, gênero, consciência, preocupação com a própria imagem, emoção, acesso à informação, confiança e hábito. Já em relação aos atributos de sustentabilidade, foram 7: Eco-friendly, garantia de origem, saúde do consumidor, fair trade, cuidado com os animais, recicláveis e ingredientes naturais.

Todos os fatores mencionados e discutidos neste artigo foram encontrados e evidenciados a partir dos artigos analisados, para então serem classificados e reorganizados em duas categorias-chave. No entanto, cabe mencionar que estudos como o de Jain e Kaur (2008) afirmam que os critérios sociodemográficos foram largamente utilizados como sendo unicamente uma forma de segmentação e identificação de perfis de consumidores de produtos sustentáveis. Desse modo, não corrobora, portanto, que esses fatores sejam utilizados como influenciadores no processo de escolha e compra.

Esse estudo apresenta limitações, pois ao mesmo tempo que o estudo proporcionou uma ampla revisão sistemática de literatura acerca do tema consumo e sustentabilidade, com 22 bases acadêmicas e practitioners pesquisadas e 95 artigos analisados com diferentes abordagens metodológicas, não foi possível realizar um estudo bibliométrico dos achados. Isso impossibilitou, por exemplo, a comparação de dados como quantidade de citação dos artigos, indexação, periódicos mais relevantes na área e, inclusive, a interrelação entre os fatores encontrados, visto a diversidade de tipos e abordagens utilizadas pelos autores dos artigos, que incluíam desde pesquisas qualitativas, quantitativas, estudos de caso e ensaios teóricos.

Pesquisas futuras poderão explicar a relação com o consumo dos atributos de sustentabilidade que não foram explicados pela literatura (que é o caso dos atributos Reutilizável, Livre de Emissões de Carbono, Agricultura Sustentável, Madeira Sustentável, Embalagem com cuidado ambiental e Eco Eficiente) ou promover um estudo macro de hierarquização de influência desses atributos no consumo, considerando sua importância e relevância, assim como a reposta das empresas em relação a essa demanda por atributos de sustentabilidade. Além disso, como sugestão de estudos futuros, a Revisão Sistemática de Literatura identificou uma incipiência de conhecimentos acadêmicos frente ao consumo de produtos sustentáveis pelo consumidor brasileiro de Classe Média, uma vez que é um tema de relevância, afinal, ao possuir maior poder de compra, essa parcela da população poderá contribuir para o aumento do consumo mundial e o conduzirá a uma maior pressão sobre os recursos que o mundo dispõe (Danciu, 2013). Considerando que o crescimento dos mercados emergentes 
de 2013 a 2020 será 3 vezes maior que os de países desenvolvidos, representando 65\% do crescimento global (Euromonitor International, 2013), pesquisas para criação de novas oportunidades de negócios nesse mercado serão relevantes. Por fim, este estudo também contribui para a elaboração futura de um arcabouço teórico e empírico que poderá servir como ponto de partida para pesquisas relacionadas à temática.

Ou seja, sem a produção sustentável, será cada vez mais difícil atender às expectativas e aspirações dos consumidores. E, mesmo se não quiserem, os consumidores terão de viver de forma mais sustentável nas próximas décadas (Danciu, 2013). A falta de conhecimento teórico na área pode ser o indício de pouca abordagem prática do tema, indicando uma oportunidade de negócio mal explorada para essa classe que, por definição, representa metade da população nacional. Como contribuição gerencial, essa lacuna teórica pode também representar uma incipiência prática de ações das empresas frente às demandas do público, que representa grande oportunidade de mercado. Pesquisas futuras podem apresentar ações que têm sido empregadas pelo meio empresarial para suprir essa necessidade de mercado, assim como melhor definir as demandas da classe neste tema.

\section{Agradecimentos}

Os autores agradecem à Coordenação de Aperfeiçoamento de Pessoal de Nível Superior - Brasil (CAPES), Código de Financiamento 001, e à Fundação Dom Cabral, pelo apoio financeiro em bolsa de pesquisa para realização deste estudo. Também agradecem as recomendações recebidas dos revisores anônimos que contribuíram para um melhor desenvolvimento das ideias aqui propostas.

\section{Referências}

Annunziata, A., \& Scarpato, D. (2014). Factors affecting consumer attitudes towards food products with sustainable attributes, Agric. Econ., 60, 353-363.

Balderjahn, I., Buerke, A., Kirchgeorg, M., Peyer, M., Seegebarth, B., \& Wiedmann, K. P. (2013). Consciousness for sustainable consumption: scale development and new insights in the economic dimension of consumers' sustainability., AMS Review, 3(4), 181-192.

Barbosa, L., \& Veloso, L. (2012, June). Consumption, Domestic Life, and Sustainability in Brazil. Global Research Forum on Sustainable Consumption and Production Workshop, Rio de Janeiro, RJ, Brazil.

Bardin, L. (1979). Análise de conteúdo. Lisboa: Edições 70.

Bardin, L. (2011). Análise de conteúdo. São Paulo: Edições 70.

Biswas, A. (2016). A Study of Consumer's willingness to pay for green products. Journal of advanced Management Science, 4(3), 211-215.

Blackwell, R. D., Miniard, P. W., \& Engel, J. F. (2005). Comportamento do Consumidor. São Paulo: Pioneira Thomson Learning. 
Carrete, L., Castaño, R., Felix, R., Centeno, E., \& González, E. (2012). Green consumer behavior in an emerging economy: confusion, credibility, and compatibility. Journal of Consumer Marketing, 29(7), 470-481.

Cunha, C. F., Spers, E. E., \& Zylbersztajn, D. (2011). Percepção sobre atributos de sustentabilidade em um varejo supermercadista. RAE - Revista de Administração de Empresas, 51(6), 542-552.

Damázio, L. F., Gonçalves, C. A., \& Shigaki, H. B. (2017). Contemporary Market Management: A Theoretical Model Proposition. In Anais, Academy of Marketing. Hull University Business School, Hull, England.

Danciu, V. (2013). The future of marketing: an appropriate response to the environment changes. Theoretical and Applied Economics, 20(5), 33-52.

Espinoza, F. S. (2004). O impacto de experiências emocionais na atitude e intenção de comportamento do consumidor. Dissertação de mestrado, Universidade Federal do Rio Grande do Sul, Porto Alegre, RS, Brasil.

Engel, J. F., Blackwell, R. D., \& Miniard, P. W. (1986). Consumer Behavior. Chicago: Dryden Press.

Euromonitor International. (2013). Reaching The Middle Classes Beyond BRIC. Euromonitor. Recuperado em 05 novembro, 2018, de: https://blog.euromonitor. com/the-emerging-middle-classes-beyond-bric/

Funaru, M., \& Baranov, A. (2012). Environmental Marketing - element for asserting the management of sustainable development. Bulletin of the Transilvania University of Braşov, 5(1), 53-58.

Green T., \& Peloza J. (2014). Finding the right shade of green: the effect of advertising appeal type on environmentally friendly consumption. Journal of Advertising, 43(2), 128-141.

Grönroos, C., \& Voima, P. (2013). Critical service logic: making sense of value creation and co-creation. Journal of the Academy of Marketing Science, 41(2), 133-150.

Gruber, V., Schlegelmilch, B. B., \& Houston, M. (2014), Inferential evaluations of sustainability attributes: exploring how consumers imply product information. Psychology and Marketing, 31(6), 440-450.

Guillen-Royo, M. (2019). Sustainable consumption and wellbeing: Does on-line shopping matter? Journal of Cleaner Production, 229, 1112-1124.

Hammerle, I. P. (2012). Bewertung von "bioPlus" Leistungen. Dissertação de Mestrado, Universität Wien, Wien, Áustria.

Hamzaoui, L. \& Zahaf, M. (2009). Exploring the decision- making process of Canadian organic food consumers: motivations and trust issues. Qualitative Market Research: An International Journal, 12(4), 443-59.

Hoek, J., Roling, N., \& Holdsworth, D. (2013). Ethical claims and labelling: An analysis of consumers' beliefs and choice behavior. Journal of Marketing Management, 29(7-8), 772-792.

Holmes, S. (2010). Firm innovations from voluntary dyadic engagement with nonprofit organisations: an exploratory UK study. Tese de doutorado, School of Management, Cranfield University, London, United Kingdom. 
Hosseinpour, M., Nezakati, H., Sidin, S. M., \& Fee, W. F. (2016). Consumer's intention of purchase sustainable products: The moderating role of attitude and trust. Journal of Marketing and Management, 7(1), 40-49.

Howard, J., \& Sheth, J. (1969). The Theory of Buyer Behavior. New York: John Wiley. Hüttel, A., Ziesemer, F., Peyer, M., \& Balderjahn, I. (2018). To purchase or not? Why consumers make economically (non-)sustainable consumption choices. Journal Of Cleaner Production, 174, 827-836.

IBGE. (2015). Indicadores de Desenvolvimento Sustentável. Recuperado em 04 novembro, 2018, de: http://www.ibge.gov.br/home/geociencias/recursosnaturais/ ids/default_2015.shtm

Iqbal, M. (2011) Sustainable Fashion Consumption and Consumer Behavior. Dissertação de mestrado, The Swedish School of Textile, University of Borås, Borås, Sweden.

Jain, S. K., \& Kaur, G. (2008). Role of Socio-demographics in segmentating and profiling green consumers. Journal of international Consumer Marketing, 18(3), 107-146.

Joshi, Y., \& Rahman, Z. (2015). Factors Affecting Green Purchase Behaviour and Future Research Directions. International Strategic Management Review, 3(1-2), 128-143.

Kalantari, K., \& Asadi, A. (2010). Designing a structural model for explaining environmental attitude and behavior of urban residents (case of Tehran). International Journal of Environmental Responsibility, 4(2), 309-20 In L. Carrete, R. Castaño, R. Felix, E. Centeno, \& E. González (2012). Green consumer behavior in an emerging economy: confusion, credibility, and compatibility. Journal of Consumer Marketing, 29(7), 470-481.

Kataria, A., Mukherjee, J., Biswas, S., \& Garg, R. (2016). An exploration of consumers' perceived value of sustainable brands in India. Asian Journal of Business Research, 6(2), 52-69.

Kostadinova, E. (2016). Sustainable Consumer Behavior: literature overview. Economic Alternatives, (2), 224-234.

Leonidou, C. N., Katsikeas, C. S., \& Morgan, N.A. (2013). "Greening" the marketing mix: do firms do it and does it pay off? Journal of the Academy of Marketing Science, 41(2), 151-170.

Lipovetsky, G. (2006). A felicidade paradoxal: ensaio sobre a sociedade do hiperconsumo. Lisboa: Edições 70.

Luchs, M. G., Naylor, R. W., Irwin, J. R., \& Raghunathan, R. (2010). The sustainability liability: potential negative effects of ethicality on product preference. Journal of Marketing, 74, 18-31.

Megicks, P., Memery, J., \& Angell, R. J. (2012). Understanding local food shopping: Unpacking the ethical dimension. Journal of Marketing Management, 28(3-4), 264-289

Monteiro, T., Giuliani, A., Zambon, M., Pizzinatto, N., \& Cunha, C. (2012). Consciência Ecológica e Atitudes dos consumidores: um estudo exploratório sobre seus impactos diante de produtos e marcas. Revista de Administração da UNIMEP, 10(3), 183-198. 
Mulrow, C. (1994). Rationale for systematic reviews. British Medical Journal, 309, 597-599.

Nespolo, D., Borelli, V. A., Fidelis, A. C. F, Machado, S. M., Olea, P. M., \& Rocha, J. M. (2016). Consumo consciente, meio ambiente e desenvolvimento sustentável: análise da tomada de decisão com base nas heurísticas. Revista Administração UFSM, 9, 137-148.

Nielsen (2015). Consumer-goods' brands that demonstrate commitment to sustainability outperform those that don't. Recuperado em 30 outubro, 2018, de: http://www.nielsen.com/eu/en/press-room/2015/consumer-goods-brands-thatdemonstrate-commitment-to-sustainability-outperform.html

Nicosia, F. M. (1966). Consumer decision processes: marketing and advertising implications. Englewood Cliffs: Prentice-Hall.

Ottman, J. A. (2012). As novas regras do Marketing verde: Estratégias, ferramentas e inspiração para o branding sustentável. São Paulo: M Books.

Ramirez, E., Jiménez, F. R., \& Gau, R. (2015). Concrete and abstract goals associated with the consumption of environmentally sustainable products. European Journal of Marketing, 49(9-10), 1645-1665.

Rettie, R., Burche, K., \& Riley, D. (2012). Normalising green behaviours: A new approach to sustainability marketing. Journal of Marketing Management, 28(3-4), 420-444.

Ribeiro, J. A., Veiga, R. T., \& Higuchi, A. K. (2016). Personality traits and sustainable consumption. Revista Brasileira de Marketing, 15(3), 297-313.

Ronchi, L., Oliveira, P. R. V., Parisotto, I. R. S., Gomes, G. (2016). A prática do consumo sustentável entre estudantes universitários. Revista metropolitana de Sustentabilidade, 6(1), 118-138.

Rothenberg, L., \& Matthews, D. (2017). Consumer decision making when purchasing eco-friendly apparel. International journal of retail \& Distribution Management, 45(4), 404-418.

Sampaio, R., \& Mancini, M. (2007). Estudos De Revisão Sistemática: Um Guia para síntese criteriosa da evidência científica. Revista Brasileira Fisioterapia, 11(1), 83-89.

Schafer, M., Jaeger-Erben, M., \& Santos, A. (2011). Leapfrogging to sustainable consumption? An explorative Green consumer behavior in an emerging economy survey of consumption habits and orientations in southern Brazil. Journal of Consumer Policy, 34(1), 175-96.

Schäufele, I., \& Hamm, U. (2017). Consumers' perceptions, preferences and willingness-to-pay for wine with sustainability characteristics: a review. Journal of Cleaner Production, 147, 379-394.

Silva, C., Gobbi, B., \& Simão, A. (2005) O uso da análise de conteúdo como uma ferramenta para a pesquisa qualitativa: descrição e aplicação do método. Organizações Rurais e Agroindustriais, 7(1). 70-81.

Silva, M. E. (2012). Consumo sustentável: a articulação de um construto sob a perspectiva do desenvolvimento sustentável. Revista Eletrônica de Ciência Administrativa, 11(2), 217-232. 
Simões, R., Giraldi, J., Oliveira, S. (2012). Influência dos valores pessoais no comportamento "verde" do consumidor. Revista portuguesa e brasileira de gestão, 11(4), 26-37.

Solomon, M. R. (2008). O comportamento do consumidor: Comprando, possuindo e sendo. São Paulo: Bookman.

Stranieri, S., Ricci, E. C., \& Banterle, A. (2017). Convenience food with environmentallysustainable atributes: a consumer perspective. Appetite, 116, 11-20.

Strategy Partners. (2010). Estratégia e Valor da Sustentabilidade (EVS®). Recuperado em 30 outubro, 2018, de: http://dom-ecc.com.br/work-view/estrategia-e-valor-dasustentabilidade/

Tranfield D., Denyer D., \& Smart P. (2003). Towards a methodology for developing evidence-informed management knowledge by means of systematic review. British Journal of Management, 14, 207-222.

Tunn, V. S. C., Bocken, N. M. P., Hende, E. A., Schoormans, J. P. L. (2019). Business models for sustainable consumption in the circular economy: An expert study. Journal of Cleaner Production, 212, 324-333.

Unruh, G., Kiron, D., Kruschwitz, N., Reeves, M., Rubel, H., \& Felde, A. (2016). Investing for a sustainable future. MIT Sloan Management Review Research Report, (57480), 1-31.

Vargo, S. L., \& Lusch, R. F. (2016). Institutions and axioms: an extension and update of service-dominant logic. Journal of the Academy of Marketing Science, 44(1), 5-23. 